\title{
Early Detection of Lung Cancer: A Statement from an Expert Panel of the Swiss University Hospitals on Lung Cancer Screening
}

\author{
T. Frauenfelder ${ }^{\mathrm{a}} \quad$ M.A. Puhan ${ }^{\mathrm{d}} \quad$ R. Lazor ${ }^{\mathrm{e}} \quad$ C. von Garnier ${ }^{f} \quad$ J. Bremerich ${ }^{\mathrm{h}}$ \\ T. Niemann $^{\text {h }}$ A. Christe ${ }^{g} \quad$ X. Montet ${ }^{i} \quad$ O. Gautschij $\quad$ W. Weder ${ }^{\text {b }}$ \\ M. Kohler ${ }^{c}$ on behalf of the expert panel \\ a Institute of Diagnostic and Interventional Radiology, ${ }^{b}$ Division of Thoracic Surgery and 'Pulmonary Division, \\ University Hospital of Zürich, and ${ }^{\mathrm{d} I n s t i t u t e}$ of Social and Preventive Medicine, Zürich University, Zürich, \\ ${ }^{e}$ Respiratory Medicine Department, Lausanne University Hospital, Lausanne, Departments of f Pulmonary Medicine \\ and ${ }^{9}$ Interventional, Pediatric and Diagnostic Radiology, University Hospital Bern, Inselspital, Bern, ${ }^{\mathrm{h}}$ Department of \\ Radiology, University Hospital of Basel, Basel, 'Department of Radiology, Geneva University Hospital and Faculty of \\ Medicine, Geneva, and 'Department of Medicine, Luzerner Kantonsspitäler, Luzern, Switzerland
}

\section{Key Words}

Early lung cancer detection · Lung cancer screening · Low-dose CT

\begin{abstract}
The discussion about setting up a program for lung cancer screening was launched with the publication of the results of the National Lung Screening Trial, which suggested reduced mortality in high-risk subjects undergoing CT screening. However, important questions about the benefit-harm balance and the details of a screening program and its cost-effectiveness remain unanswered. A panel of specialists in chest radiology, respiratory medicine, epidemiology, and thoracic surgery representing all Swiss university hospitals prepared this joint statement following several meetings. The panel argues that premature and uncontrolled introduction of a lung cancer screening program may cause substantial harm that may remain undetected without rigorous quality control. This position paper focuses on the requirements of running such a program with the objective of harmonizing efforts across the involved specialties and institutions and
\end{abstract}

\section{KARGER}

E-Mail karger@karger.com www.karger.com/res defining quality standards. The underlying statement includes information on current evidence for a reduction in mortality with lung cancer screening and the potential epidemiologic implications of such a program in Switzerland. Furthermore, requirements for lung cancer screening centers are defined, and recommendations for both the CT technique and the algorithm for lung nodule assessment are provided. In addition, related issues such as patient management, registry, and funding are addressed. Based on the current state of the knowledge, the panel concludes that lung cancer screening in Switzerland should be undertaken exclusively within a national observational study in order to provide answers to several critical questions before considering broad population-based screening for lung cancer.

(c) 2014 S. Karger AG, Basel

\section{Introduction}

Recently published data from the National Lung Screening Trial (NLST) showed a $20 \%$ reduction in lung cancer mortality in subjects who underwent CT screen-
C 2014 S. Karger AG, Basel

0025-7931/14/0873-0254\$39.50/0
T. Frauenfelder

Institute of Diagnostic and Interventional Radiology

University Hospital of Zürich, Rämistrasse 100

CH-8091 Zürich (Switzerland)

E-Mail thomas.frauenfelder@usz.ch 
Table 1. Potential benefits and harms of lung cancer screening

\begin{tabular}{ll}
\hline Benefits & Harms \\
\hline Mortality reduction & $\begin{array}{l}\text { Unnecessary diagnostic procedures and procedure-related complications } \\
\text { (due to the high number of false-positive findings) }\end{array}$ \\
\hline $\begin{array}{l}\text { Improved quality of life } \\
\text { (in case of early detection) }\end{array}$ & Radiation exposure \\
\hline Smoking cessation & $\begin{array}{l}\text { Overdiagnosis } \\
\text { Anxiety and impaired quality of life (due to false-positive results) } \\
\text { False sense of reassurance and thus continuation of smoking } \\
\text { (in case of a normal CT scan) }\end{array}$ \\
\hline
\end{tabular}

ing compared to those randomized to conventional chest $\mathrm{X}$-ray [1]. In Europe, several randomized controlled studies using low-dose CT (LDCT) as a screening tool for the detection of lung cancer have been or are still being performed [2-7]. The largest among these European randomized LDCT screening trials, i.e. the Dutch-Belgian NELSON Trial, started in 2003 and the final results are awaited $[5,6]$.

Several associations and panels released statements on whether or not to implement lung cancer screening in clinical practice after the publication of the results of the NLST [8-13]. Some of these statements highlighted the need for further improvement of CT screening and describe specific areas which should be addressed in guidelines and recommendations [11, 14-16]. In contrast, other associations suggested that screening should be immediately implemented [17, 18]. Despite the positive results of the NLST, we think that the following points need to be established before large-scale lung cancer screening programs with LDCT can be implemented in the clinical routine: the definition of the population at risk, the variability of radiological standards and adapted guidelines for use in national screening programs, the number and frequency of screening rounds, the additional value of lung nodule volumetry versus $2-\mathrm{D}$ measurement, the development of appropriate guidelines for the clinical workup of 'indeterminate nodules' resulting from CT screening programs, the quality of life including the psychological consequences of taking part in such a program, the role of smoking cessation intervention, and questions related to costs and cost-effectiveness $[15,19]$.

The discussion about lung cancer screening has also started in Switzerland [20]. The SUVA (Schweizerische Unfallversicherungsanstalt), an independent, nonprofit company under public law, started a screening program for patients with a history of asbestos exposure shortly after the publication of the results of the NLST although there is no evidence from randomized controlled trials that lung cancer screening is effective in such a population. This program involves private physicians and is running in many radiology departments without any quality control. Other private institutes have also started to advertise lung cancer screening. In June 2012, a first meeting of several stakeholders representing the Swiss university hospitals took place in an attempt to constitute a national expert panel to address open questions related to lung cancer screening programs. The expert panel prepared this statement paper on the topic, mainly focusing on practical issues related to lung cancer screening with LDCT, with the awareness that smoking cessation is the most efficient method to reduce the number of lung cancer cases. Other important issues such as alternative methods of lung cancer screening and the implications of screening for therapeutic decisions or socioeconomic aspects are only briefly or not addressed. The panel agreed that, at present, lung cancer screening should be performed exclusively in the framework of an observational study accompanied by a prospective registry to ensure quality control and to generate longitudinal observational data on aspects of screening-related harm (table 1) and to assess the feasibility, the quality, and especially the direct and indirect costs and cost-effectiveness of such an intervention.

The purpose of this paper is to provide information on how such a study on lung cancer screening with LDCT should be implemented in Switzerland. At this point, it seems important to harmonize efforts across the involved specialties and institutions, to define quality standards, and to develop the appropriate study methodology. 


\section{Epidemiology and Effectiveness of Lung Cancer Screening Programs}

Worldwide, lung cancer is the most commonly diagnosed cancer (12.7\% of all cancers) and, because of its high fatality rate, the most common cause of death from cancer [21]. Poor 5-year survival rates (16\%) are related to advanced disease at diagnosis [30\% Union for International Cancer Control (UICC) stage III and 40\% stage IV] [22]. In Switzerland, annually 2,500 men (13\% of all cancers) and 1,700 women ( $8 \%$ of all cancers) are diagnosed with lung cancer and 2,000 men (23\% of all cancer deaths) and 900 women (13\% of all cancer deaths) die from lung cancer [23]. While in men the incidence rate declined in Switzerland between the 1980s and 2007, the incidence of lung cancer in women almost doubled within the same time span and is now reaching a plateau.

Earlier studies showed that lung cancer screening studies with conventional chest X-ray and sputum cytology detected more lung cancers, earlier cancer stages, and smaller tumors, but there was no reduction in late-stage tumors and lung cancer-specific mortality [16, 24-35]. With its introduction approximately one decade ago, LDCT enhanced the detection of lung cancer [36].

According to a recent systematic review that analyzed the data of 8 randomized controlled trials and 13 cohort studies to assess the possible benefits and harms of lung cancer screening, lung cancer-specific mortality was only reported in 4 trials (Detection and Screening for Early Lung Cancer by Novel Imaging Technology and Molecular Assays (DANTE) [2], NLST [1], Danish Lung Cancer Screening Trial (DLCST) [4], and MILD Trial [7]) [16]. Of these, the NLST randomized 53,439 smokers and exsmokers aged 55-74 years to an annual conventional chest X-ray or LDCT over 3 years [1]. After a median follow-up duration of 6.5 years, the NLST found a reduction in lung cancer-related mortality of $20 \%$ in the LDCT screening group compared to the chest $\mathrm{X}$-ray group. The authors calculated that 320 individuals needed to be screened by LDCT to prevent 1 lung cancer-related death. In contrast, the smaller DLCST [4] and the DANTE Trial [2] found no significant mortality reduction with 5 annual rounds of LDCT compared to the usual care at a median follow-up of 34 and 58 months, respectively. Furthermore, no statistically significant positive or negative effects on smoking behavior have been observed in any of the LDCT lung cancer screening trials to date.

The radiation exposure of a single LDCT examination is about 4- to 5-fold and 9- to 10 -fold lower than the values of conventional chest CT and PET-CT, respectively
$[37,38]$. However, a screening program with several LDCT examinations may substantially increase the cumulative radiation exposure; for example, an estimated average of $8 \mathrm{mSv}$ was delivered in the NLST due to screening and diagnostic investigations $[1,16]$. Using exposure models, Bach et al. [16] estimated that radiation exposure from the NLST would result in 1 cancer death for every 2,500 individuals screened. The authors concluded that in the NLST population the decrease in mortality due to screening outweighed the risk of radiation, but in individuals younger than 42 years or in those at a lower risk for lung cancer this may not be the case [16, 39]. According to the calculation of Bach et al. [16], the cumulative 10-year risk for the diagnosis of lung cancer in participants meeting the minimum entry criteria and based on the assumption that they had quit smoking at the time of study entry was approximately $2 \%$ for the NLST. Although screening programs may improve the quality of life thanks to the reduced lung cancer-related morbidity associated with advanced disease, they may also generate anxiety and discomfort, thus potentially causing harm [16]. The Dutch-Belgian NELSON Trial included 15,822 subjects. Half of them were randomized to the LDCT arm. Screening was performed at baseline and 1,3 , and 5.5 years later (4 rounds) with a 10 -year follow-up period $[5,6]$. In a subgroup analysis of 351 participants, 87-99\% (depending on the specific questionnaire used) of individuals experienced no discomfort due to CT, but $46 \%$ were distressed awaiting the CT results [40].

\section{Screening Population}

Definition of the population to be screened is a challenging task and requires careful balancing of the benefits and harms of a screening program. As discussed above, the benefit/harm balance depends substantially on the absolute risk of these outcomes. Therefore, lung cancer screening programs should focus, like other screening programs, on the population at increased risk for the disease to be prevented and exclude people in which the benefit-harm balance is unlikely to be favorable (e.g. a lung cancer screening program in people with no smoking history or less than 10 pack-years where the number needed to screen would be too high). For example, the NLST focused on subjects between 55 and 74 years of age with a cigarette smoking history of at least 30 pack-years without evidence of lung cancer (e.g. hemoptysis or unexplained weight loss in the preceding year). The Early Lung 
Cancer Action Project (ELCAP) defined its population similarly [41]. In the NLST population, the number needed to screen to prevent 1 lung cancer death out of 320 persons undergoing 3 annual CT scans compared to no CT scans [1] needs to be contrasted with the harm in this population. The NLST reported overall mortality and major complication rates after diagnostic procedures for benign nodules in the LDCT group of 4.1 and 4.5 per 10,000 participants, respectively, compared to 1.1 and 1.5 per 10,000 in the chest X-ray group $[1,16]$. Additionally, screening may identify lung cancer that would not have affected the patient's life if left untreated, a phenomenon termed overdiagnosis [42]. Bach et al. [43] addressed the problem of overdiagnosis in 2007 using calculations based on CT screening. They showed that, despite the fact that more tumors were detected by CT screening, the numbers of advanced tumors and tumor related deaths were not reduced. Other studies based on X-rays as well as one recent study on LDCT have reported an overdiagnosis rate of about $25 \%[16,44-46]$. The findings of the NLST suggested that overdiagnosis might have occurred, but due to the study design this cannot be ascertained and quantified at this stage.

Evidence of both the benefits and the harms of early lung cancer detection programs is still scarce and therefore it is currently difficult to define a population other than the one defined by the NLST in which the benefitharm balance of lung cancer screening is likely to be favorable. A recent study by Kovalchik et al. [47] analyzed the NLST population concerning their risk of developing lung cancer and showed that in subjects with multiple risk factors the benefit clearly outweighs the harm. The findings of this analysis possibly call for individual risk stratification in lung cancer screening programs, which is likely to improve the cost-effectiveness of LDCT screening [47]. Also, the most appropriate number of screening rounds is uncertain at this stage. Therefore, we suggest that, if screening is performed, the screened population should be the same as in the NLST, focusing on individuals aged 55-74 years with at least 30 pack-years of smoking history, either active or discontinued for less than 15 years, with no clinical symptoms of lung cancer. The recommendation of extending the screened population to other age groups, to patients with COPD, to those with a previous history of lung cancer, or to those with a lower level of smoking exposure [17] is not supported by the current evidence, it is likely to be harmful, and it should therefore be avoided. Recent studies suggest that the selection criteria for lung cancer screening can be refined [48].

Early Detection of Lung Cancer

\section{Minimal Requirements of a Lung Cancer Screening Center}

Performed within a national prospective trial or registry, we propose that lung cancer screening should only be done at institutions with the necessary infrastructure and expertise for the multidisciplinary workup of lung nodules and management of lung cancer, which are most likely to replicate the results of the NLST $[14,15,49,50]$. These requirements should be enforced by the health authorities similar to the screening program for breast cancer and should adhere to standards for training, infrastructure, procedures, and documentation including the following:

- A chest imaging unit with low-dose HRCT (for the minimum technical requirements, see Technique and Algorithm of Lung Nodule Assessment), percutaneous CT-guided needle biopsy, and PET-CT

- A respiratory medicine unit with state-of-the-art cardiopulmonary exercise testing facilities and video bronchoscopy

- A cytopathology department

- A thoracic surgery unit experienced in minimally invasive surgical techniques

- Comprehensive pre- and postoperative care, including an intensive care unit and pulmonary rehabilitation

- An oncology unit with organ-specific multidisciplinary tumor boards on a routine basis

- A smoking cessation program and counseling

All specialists involved should be board certified in their respective disciplines.

Patient information on lung cancer screening should be provided both orally and in written form with the help of specific information material. The patients are assessed according to the NLST criteria, which does not provide an explicit risk prediction for lung cancer but restricts the screening to a population at increased risk for lung cancer compared to subjects who, for example, smoked less or are outside of the age range used in the NLST $[47,50,51]$. The information material should provide key data about the expected screening benefits and harms in a neutral and nonpersuasive way [14], including an explanation of the difference between a screening test and a diagnostic procedure, the facts that screening will not detect all cancers, that screening is more likely to detect a benign abnormality than cancer, and that the benefit-harm ratio is still unclear, and the potential risks of invasive diagnostic and therapeutic procedures $[13,15]$. A flowchart may be provided showing the possible scenarios [49]. Sufficient time should be allowed for decision making and the first screening CT should not be performed on the same day 
the information is provided. Smoking cessation programs are to be systematically integrated. After a negative screening round, patients should be informed of the symptoms of lung cancer [15].

Institutions performing lung cancer screening should adopt an ethical chart stating that they do not recruit patients through direct or indirect advertisement, use fear of cancer to promote lung cancer screening, let patients believe that the cancer risk can be eliminated by screening, or offer screening at reduced costs with the aim of generating profit from additional diagnostic and therapeutic procedures [50].

\section{Technique and Algorithm of Lung Nodule Assessment}

\section{Imaging Protocol}

For lung cancer screening, at best a 64-slice multidetector CT scanner or a later generation scanner should be used. This is to achieve the optimal spatial resolution for accurate and reproducible measurements of small nodules. The slice thickness should be $1.5 \mathrm{~mm}$ or less and imaging has to be performed without contrast in full inspiration. A low-dose protocol must be used with an effective dose of around $1 \mathrm{mSv}$ depending on the patient's weight and including iterative reconstruction whenever possible.

\section{Lung Nodule Assessment}

A lung nodule is defined as a small, approximately spherical, nonlinear, circumscribed focus of abnormal soft tissue $[52,53]$. Lung nodules can be categorized as calcified nodules in the presence of a benign pattern of calcium, assolid nodules if they have areas of homogeneous soft tissue attenuation, as partly solid nodules if they have both solid and ground-glass attenuation, and as nonsolid (pure ground-glass) nodules if they only have ground-glass attenuation. Although the shape of a solid lung nodule may add to the evaluation regarding its potential malignancy, the size or volume of the nodule should preferably be used as the objective criteria [5456].

The expert panel proposes employment of the algorithm outlined in the statement of the National Comprehensive Cancer Network (NCCN) for the management of solid nodules and partly/nonsolid nodules [9]. In case of new nodules detected at the annual or follow-up LDCT, the algorithm for the management of solid nodules and partly/nonsolid nodules should be applied in cases of no suspected infection. The workup for solid nodules should be in accordance with the Fleischner Society criteria [57]. There are new recommendations from the Fleischner Society regarding the management of subsolid nodules, which still need to be integrated into the current algorithm [58]. For nonsolid nodules, the guidelines follow the recommendation of Godoy and Naidich [59]. Although in all running screening programs size is the most important factor, results from the NELSON Trial, for example, show that perifissural nodules are mostly benign and do not need further workup even if they are growing $[54,60]$. In cases of suspected infection, a follow-up LDCT should be considered after 6-8 weeks and if the nodules increase in size a diagnostic workup is necessary.

Though all of these algorithms utilize the mean nodule diameter as the only relevant criterion, the largest European lung cancer screening trial, i.e. the NELSON Trial, focused on the volume and the change in volume as the major criteria for lung nodule assessment and further management [6]. French experts of the French intergroup (IFCT) and the Groupe d'Oncologie De Langue Française (GOLF) also adopted such an approach [10]. The volumetric analysis may allow a more accurate and more differentiated assessment of lung nodules. The preliminary results of the NELSON Trial suggest that volumetry of the nodule may be a useful parameter to reduce the false-positive and false-negative rates, but this technology is not widely available yet [6]. Nevertheless, the panel advocates the use of lung nodule volumetry in the case of a standardized CT protocol and use of the same software for each measurement.

Computer-assisted detection (CAD) of lung nodules has recently become more important. Several studies have shown that the use of CAD in radiological practice can significantly improve the diagnostic accuracy of pulmonary nodule detection $[61,62]$. In an analysis based on data from the NELSON Trial, the sensitivity of CAD to detect lung nodules was $96.7 \%$ compared to $78.1 \%$ for conventional double reading without CAD [61]. This difference seems to be particularly explained by the observation that nodules attached to vessels are often missed by conventional reading. Therefore, the panel advocates the use of a CAD system as a second (or additional) reader in a lung cancer screening program. No benefit has been shown for double reading [63].

Workup of Solid Nodules. If malignancy is highly probable, then the nodule should be surgically removed. PET$\mathrm{CT}$ as a single method has a negative predictive value of only $81 \%$ as shown by van't Westeinde et al. [64], which is insufficient to rule out malignancy. However, the findings 
of other studies suggest that PET-CT can be used to reduce the number of false-positive findings, especially when used in combination with other diagnostic procedures [65, 66]. Accordingly, Ashraf et al. [66] found that the use of both PET and the volume doubling time increases the sensitivity and specificity for lung cancer diagnosis to 90 and $82 \%$, respectively.

Workup of Subsolid Nodules. The management of subsolid nodules should be based on the recommendations of the Fleischner Society [58]. As shown in the MILD Trial, the growth rate of subsolid nodules is usually very low, and thus active prolonged surveillance is recommended [67]. This may be adapted according to the type of subsolid nodule, the initial nodule size, and the past history of lung cancer [68]. In fast growing ground-glass lesions, PET has rarely been proven to predict malignancy, and thus surgery is often the diagnostic strategy of choice [55, $59,69]$.

Besides lung cancer-related mortality, the NLST also found a $6.7 \%$ decrease in all-cause mortality [1]. There are several pathologies that may be found when an LDCT is being performed and this may affect mortality. Graham et al. [70], for example, showed that a diagnosis of severe coronary calcification has a significant impact on mortality. It is therefore possible that analysis of imaging features other than lung nodules may have an impact on survival or other clinical outcomes. The relevance of these additional findings has been investigated by several groups [71-73]. In all of these studies the number of significant incidental findings did not exceed $7 \%$. This was influenced by the wider scan range in that population, covering parts of the upper abdomen [72]. Priola et al. [72] estimated that the additional incurring costs might not be extensive. However, currently there is no consensus on whether or not these incidental findings should be reported. This has also been discussed based on data of the NELSON Trial, and van de Wiel et al. [71] advised against systematic reporting of all incidental findings.

\section{Patient Management}

\section{Infrastructural Needs and Organization}

The individual management of patients participating in a lung cancer screening study is of crucial importance. Dedicated, well-trained medical and paramedical staff embedded within a well-organized infrastructure is the key to running an efficient lung cancer screening program. The required infrastructure for such a center as well as how participants should be informed is discussed in more detail in Minimal Requirements of a Lung Cancer Screening Center.

The core facility for patient management should be a dedicated outpatient clinic within a chest unit to guarantee high-quality patient counseling and treatment. In addition, the patient flow and the interaction between the several involved medical specialties and the referring GP are key issues that need to be precisely defined in written protocols. Figure 1 summarizes a possible workflow for the management of patients.

\section{Interaction between Radiologists, Pulmonologists, and} Other Specialists

Interaction between specialists becomes important if a CT scan is abnormal. In weekly interdisciplinary meetings (radiology, pulmonary medicine, and thoracic surgery) any pathological CT finding requiring further investigation should be discussed. Lung nodules for which a CT follow-up is planned within the screening process do not necessarily need interdisciplinary assessment.

The joint decision of the interdisciplinary assessment team on the necessary further diagnostic or therapeutic steps should be documented in writing and constitute the basis upon which the chest unit discusses the findings of the CT scan with the patient.

\section{Communication of Pathological Findings to the}

Patient, Coordination of Further Diagnostic Steps,

and Communication with the Referring Physician

The chest unit should discuss all pathological findings described by the radiologist (this also includes extrathoracic findings) with the patient during a medical appointment. A letter detailing the results of the CT scan and recommendations regarding further investigations and/ or follow-up CT screening should be forwarded to the referring physician, the patient, and members of the interdisciplinary assessment team. The chest unit may organize further investigations. It is important that the chest unit play an active role in the management of the patient in accordance with the referring physician.

\section{Smoking Cessation}

All participating patients should be advised to cease smoking and they should be actively encouraged to participate in a structured smoking cessation program. Counseling and referral should be repetitiously offered to the participant whenever she/he is seen in the chest unit. Smoking cessation programs should follow international recommendations, for instance those of the Centers for Disease Control and Prevention [74]. 
Fig. 1. Summary of a possible workflow for the management of patients.
The patient is referred for CT screening to the chest unit by the GP (web-based form which includes the criteria for screening eligibility).

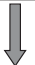

The chest unit sees the patient, gives advice to the patient regarding smoking cessation explains the potential harms and benefits of the $\mathrm{CT}$, and obtains informed consent. The chest unit books a CT appointment and forwards the electronic file to the database.

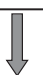

The institute of radiology performs the CT scan. The patient is dismissed home without discussing the results of the $\mathrm{CT}$ scan (exception: any disease requiring immediate action).

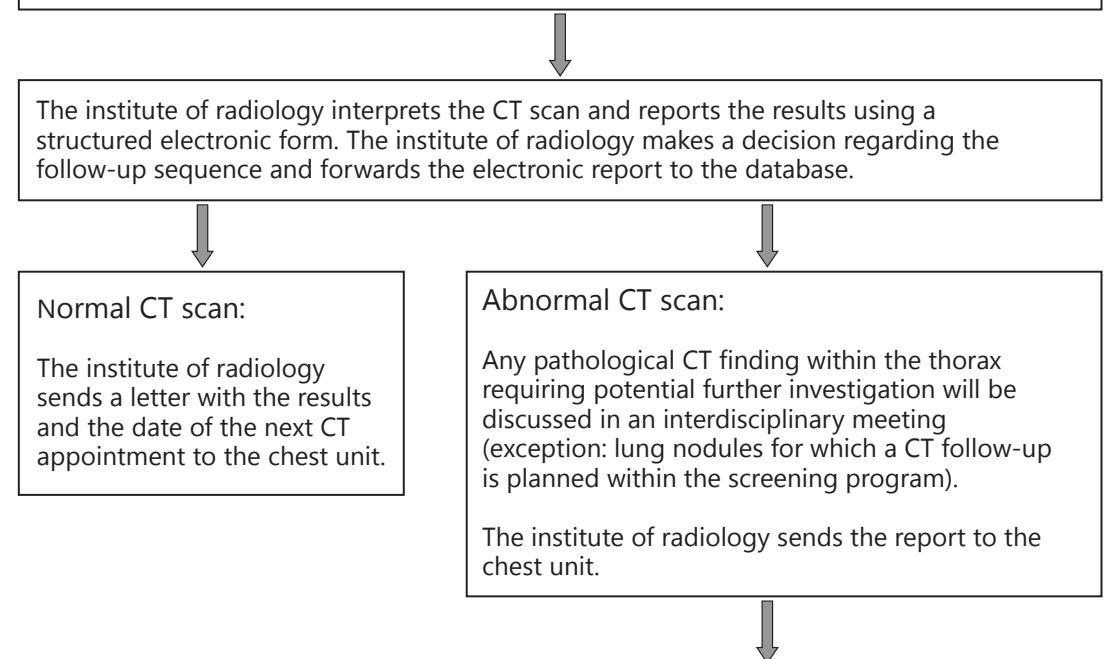

The chest unit sends a letter with the appointment date to the patient.

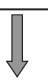

The chest unit explains the CT findings to the patient. The chest unit sends a letter with the results and recommendations (including the next CT appointment) to the GP, the patient, and the members of the interdisciplinary assessment team. The chest unit organizes further investigations if needed.

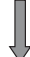

The chest unit forwards the electronic form with the decision and recommendations to the database.

\section{Costs and Cost-Effectiveness}

In the NLST, 320 patients needed to be screened to avoid 1 cancer death. McMahon et al. [75] assessed the cost-effectiveness of the NLST comparing the estimated quality-adjusted life years (QALY; measurement of the disease burden, including the quality and quantity of life lived) for lung cancers based on the screening test to either no intervention or the addition of a smoking cessation program for both study arms [75]. In the latter pub- lication, the calculated costs ranged between USD 126,000 and USD 169,000 per QALY for current and former smokers aged 50-74 years with a smoking history of at least 20 pack-years. If only subjects with at least 40 packyears were included in the analysis, the costs were reduced to a range between USD 110,000 and USD 166,000 per QALY. If linked to a smoking cessation program with an assumed background cessation rate of $6 \%$, the costs were estimated to be USD 75,000 per QALY for subjects aged $\geq 50$ years and at least a 20 -pack-year smoking his- 
tory. A comparison of these data with the cost of colorectal screening (USD 13,000-32,000 per QALY) or breast cancer screening by mammography (USD 47,700 per QALY) implies that the population to be included in a lung cancer screening program needs to be defined very carefully. Another study by Goulart et al. [76] calculated that LDCT screening will avoid 8,100 premature deaths from lung cancer at a cost of USD 1.3-2 billion in annual national health care expenditures in the USA. Thus, the additional cost of screening to avoid 1 death would be USD 240,000. In summary, to date no study has been able to show that LDCT screening is cost-effective. Further analysis of the NLST may determine whether lung screening is cost-effective; however, these estimations largely depend on the parameters and assumptions used. For example, Pyenson et al. [77] calculated that the cost per life year saved would be below USD 19,000, which is comparable to the cost of mammography screening (USD $18,000)$.

The cost of an LDCT and 2 outpatient appointments is approximately CHF 270-350 and CHF 250, respectively, based on the Swiss health tax system (TARMED), thus resulting in a direct cost of one screening round of approximately CHF 520-600. However, these direct costs are only a fraction of the total incurring costs, as the diagnostic workup and treatment related-costs cannot be reliably predicted from the available data. It is therefore crucial to gain more knowledge of these costs, not only for the health authorities but also for insurance providers.

\section{Call for a National Registry}

Quality maintenance is the ongoing process of establishing and improving the standards for all components of a program. In a multidisciplinary setting, monitoring of quality is critical for the success of a lung cancer screening program. Comparison of practices and outcomes between screening centers is needed to guarantee highquality standards at a national level. We therefore suggest setting up a common national registry with the following goals:

- To characterize the population that undergoes lung cancer screening. This should ensure that only eligible subjects are included and not those who do not, according to the current evidence, qualify for the program.

- To monitor the adherence to quality standards for all steps of the program. Quality indicators should be col- lected for the initial encounter where subjects are informed about the potential harms, for the screening CTs, for the invitation and adherence to a smoking cessation program, and for the follow-up diagnostic and therapeutic procedures that follow the screening CTs.

- To collect short-, mid-, and long-term data on benefit and harm outcomes.

- To evaluate the costs and cost-effectiveness not only of the screening procedure itself but also of all consecutive costs.

Such a registry is a central element for quality assurance and would provide a basis for detecting deviations from standardized procedures and a comparison of outcomes with those of the NLST and other studies. Also, comparisons of quality and outcomes across centers and over time need to be performed. These data would be required to inform the decision of whether and how to implement a lung cancer screening program in Switzerland, as well as for discussions with legal authorities and health insurances. However, as the proposed study design would be a purely observational study without a control group, it will not be possible to assess the efficacy of CT screening regarding the reduction of lung cancer mortality.

\section{Conclusion}

Lung cancer is a lethal disease associated with substantial medical and economic burdens. The NLST is currently the only randomized trial that has shown that lung cancer screening may reduce mortality, and the results of the NELSON Trial are eagerly awaited. The possible advantage of LDCT screening has to be balanced against the potential of inducing harm. Despite the benefit of early lung cancer detection and the prevention of tumor-related deaths, LDCT screening may produce harm, e.g. through unnecessary biopsies, radiation exposure, and psychosocial stress. Before lung cancer screening can be broadly implemented in clinical practice in Switzerland, these critical issues have to be addressed appropriately. Therefore, based on the current evidence it seems important to stress that lung cancer screening should be exclusively performed in the setting of an observational study organized by skilled professionals, in institutions with the appropriate infrastructure and expertise, and based on recommendations set forth by harmonized guidelines and standardized procedures. 


\section{Acknowledgements}

The following experts were part of the committee and contributed to this statement: T. Frauenfelder, Institute of Diagnostic Radiology, University Hospital of Zürich, Zürich, Switzerland; M. Puhan, Institute of Social and Preventive Medicine, Zürich University, Zürich, Switzerland; M. Kohler, Pulmonary Division, University Hospital of Zürich, Zürich, Switzerland; W. Weder, Division of ThoracicSurgery, University Hospital ofZürich,Zürich,Switzerland; A. Christe, Department of Interventional, Pediatric and Diagnostic Radiology, Inselspital, University Hospital Bern, Bern, Switzerland; J.T. Heverhagen, Department of Interventional, Pediatric and Diagnostic Radiology, Inselspital, University Hospital Bern, Bern, Switzerland; C. Von Garnier, Department of Pulmonary Medicine, Inselspital, University Hospital Bern, Bern, Switzerland; T. Geiser, Department of Pulmonary Medicine, Inselspital, University Hospital Bern, Bern, Switzerland; T. Niemann, Department of Radiology, University Hospital of Basel, Basel, Switzerland; J. Bremerich, Department of Radiology, University Hospital of Basel, Basel, Switzerland; R. Lazor, Respiratory Medicine Department, Lausanne
University Hospital (CHUV), Lausanne, Switzerland; C. Beigelmann, Department of Radiodiagnosis and Interventional Radiolgy, Lausanne University Hospital (CHUV), Lausanne, Switzerland; F. Levy, Cancer Epidemiology Unit and Registre Vaudois des Tumeurs, Institute of Social and Preventive Medicine (IUMSP), Lausanne University Hospital (CHUV), Lausanne, Switzerland; X. Montet, Department of Radiology, Geneva University Hospital and Faculty of Medicine, Geneva, Switzerland; O. Gautschi, Department of Medicine, Luzerner Kantonsspital, Luzern, Switzerland (representing the SAKK Lung Group), and M. Brutsche, Division of Pneumology, Kantonsspital, St. Gallen, Switzerland (representing the Schweizerische Krebsliga).

This statement was reviewed by: L.P. Nicod, Head of the Respiratory Medicine Department, Lausanne University Hospital (CHUV), Lausanne, Switzerland; H.B. Ris, Head of the Thoracic Surgery Department, Lausanne University Hospital (CHUV), Lausanne, Switzerland; N. Howarth, Radiology, Clinique des Grangettes, Geneva, Switzerland, and P. Gasche, Department of Internal Medicine, Division of Pulmonary Medicine, University Hospital of Geneva, Geneva, Switzerland.

\section{References}

1 Aberle DR, Adams AM, Berg CD, Black WC, Clapp JD, Fagerstrom RM, et al: Reduced lung-cancer mortality with low-dose computed tomographic screening. N Engl J Med 2011;365:395-409.

- Infante M, Cavuto S, Lutman FR, Brambilla G, Chiesa G, Ceresoli G, et al: A randomized study of lung cancer screening with spiral computed tomography: three-year results from the DANTE trial. Am J Respir Crit Care Med 2009;180:445-453.

3 Baldwin DR, Duffy SW, Wald NJ, Page R, Hansell DM, Field JK: UK Lung Screen (UKLS) nodule management protocol: modelling of a single screen randomised controlled trial of low-dose CT screening for lung cancer. Thorax 2011;66:308-313.

4 Saghir Z, Dirksen A, Ashraf H, Bach KS, Brodersen J, Clementsen PF, et al: CT screening for lung cancer brings forward early disease: the randomised Danish Lung Cancer Screening Trial - status after five annual screening rounds with low-dose CT. Thorax 2012;67:296-301.

5 van Iersel CA, de Koning HJ, Draisma G, Mali WP, Scholten ET, Nackaerts K, et al: Riskbased selection from the general population in a screening trial: selection criteria, recruitment and power for the Dutch-Belgian randomised lung cancer multi-slice CT screening trial (NELSON). Int J Cancer 2007;120:868874.

6 van Klaveren RJ, Oudkerk M, Prokop M, Scholten ET, Nackaerts K, Vernhout R, et al: Management of lung nodules detected by volume CT scanning. N Engl J Med 2009;361: 2221-2229.

7 Pastorino U, Rossi M, Rosato V, Marchiano A, Sverzellati N, Morosi C, et al: Annual or biennial CT screening versus observation in heavy smokers: 5 -year results of the MILD trial. Eur J Cancer Prev 2012;21:308-315.

8 Jaklitsch MT, Jacobson FL, Austin JH, Field JK, Jett JR, Keshavjee S, et al: The American Association for Thoracic Surgery guidelines for lung cancer screening using lowdose computed tomography scans for lung cancer survivors and other high-risk groups. J Thorac Cardiovasc Surg 2012;144: 33-38.

9 National Comprehensive Cancer Network: Clinical Practice Guidelines in Oncology: Lung Cancer Screening Version 1. 2012. http:// www.nccn.org/professionals/physician_gls/ pdf/lung_screening.pdf.

$\checkmark 10$ Couraud S, Cortot AB, Greillier L, Gounant V, Mennecier B, Girard N, et al: From randomized trials to the clinic: is it time to implement individual lung-cancer screening in clinical practice? A multidisciplinary statement from French experts on behalf of the French intergroup (IFCT) and the Groupe d'Oncologie de Langue Francaise (GOLF). Ann Oncol 2013;24:586-597.

11 Detterbeck FC, Mazzone PJ, Naidich DP, Bach PB: Screening for lung cancer: diagnosis and management of lung cancer, 3rd ed American College of Chest Physicians evidence-based clinical practice guidelines. Chest 2013;143:e78S-e92S.

12 Smith RA, Brooks D, Cokkinides V, Saslow D, Brawley OW: Cancer screening in the United States, 2013: a review of current American Cancer Society guidelines, current issues in cancer screening, and new guidance on cervical cancer screening and lung cancer screening. CA Cancer J Clin 2013;63:88-105.
13 Boiselle PM, Lynm C, Livingston EH: JAMA patient page: lung cancer screening. JAMA 2013;309:1948.

14 Field JK, Baldwin D, Brain K, Devaraj A, Eisen T, Duffy SW, et al: CT screening for lung cancer in the UK: position statement by UKLS investigators following the NLST report. Tho$\operatorname{rax} 2011 ; 66: 736-737$.

15 Field JK, Smith RA, Aberle DR, Oudkerk M, Baldwin DR, Yankelevitz D, et al: International Association for the Study of Lung Cancer Computed Tomography Screening Workshop 2011 report. J Thorac Oncol 2012;7:10-19.

16 Bach PB, Mirkin JN, Oliver TK, Azzoli CG, Berry DA, Brawley OW, et al: Benefits and harms of CT screening for lung cancer: a systematic review. JAMA 2012;307:2418-2429.

17 Jacobson FL, Austin JH, Field JK, Jett JR, Keshavjee S, MacMahon H, et al: Development of the American Association for Thoracic Surgery guidelines for low-dose computed tomography scans to screen for lung cancer in North America: recommendations of the American Association for Thoracic Surgery Task Force for Lung Cancer Screening and Surveillance. J Thorac Cardiovasc Surg 2012; 144:25-32.

18 Vansteenkiste J, De Ruysscher D, Eberhardt WE, Lim E, Senan S, Felip E, et al: Early and locally advanced non-small-cell lung cancer (NSCLC): ESMO clinical practice guidelines for diagnosis, treatment and follow-up. Ann Oncol 2013;24:vi89-vi98.

19 Vansteenkiste J, Dooms C, Mascaux C, Nackaerts K: Screening and early detection of lung cancer. Ann Oncol 2012;23:x320-x327.

20 Russi EW: Lung cancer screening has the potential to safe lives, but shall we do it? Swiss Med Wkly 2011;141:w13185. 
-21 Ferlay J, Shin HR, Bray F, Forman D, Mathers C, Parkin DM: Estimates of worldwide burden of cancer in 2008: GLOBOCAN 2008. Int J Cancer 2010;127:2893-2917.

22 Howlader N, Noone AM, Krapcho M, Neyman N, Aminou R, Waldron W, et al: SEER Cancer Statistics Review, 1975-2008. Bethesda, National Cancer Institute, 2011. http:// seer.cancer.gov/csr/1975_2008/.

23 Bouchardy C, Lutz J, Kühni C: Krebs in der Schweiz, Stand und Entwicklung von 1983 bis 2007. Neuchâtel, NICER und Bundesamt für Statistik, 2011.

-24 Tockman MS, Erozan YS, Gupta P, Piantadosi S, Mulshine JL, Ruckdeschel JC: The early detection of second primary lung cancers by sputum immunostaining. LCEWDG Investigators. Lung Cancer Early Detection Group. Chest 1994;106:385S-390S.

25 Tockman MS, Mulshine JL: Sputum screening by quantitative microscopy: a new dawn for detection of lung cancer? Mayo Clin Proc 1997;72:788-790.

26 Prorok PC, Andriole GL, Bresalier RS, Buys SS, Chia D, Crawford ED, et al: Design of the Prostate, Lung, Colorectal and Ovarian (PLCO) Cancer Screening Trial. Control Clin Trials 2000;21:273S-309S.

-27 Payne PW, Sebo TJ, Doudkine A, Garner D, MacAulay C, Lam S, et al: Sputum screening by quantitative microscopy: a reexamination of a portion of the National Cancer Institute Cooperative Early Lung Cancer Study. Mayo Clin Proc 1997;72:697-704.

28 Palmisano WA, Divine KK, Saccomanno G, Gilliland FD, Baylin SB, Herman JG, et al: Predicting lung cancer by detecting aberrant promoter methylation in sputum. Cancer Res 2000;60:5954-5958.

29 Miozzo M, Sozzi G, Musso K, Pilotti S, Incarbone M, Pastorino U, et al: Microsatellite alterations in bronchial and sputum specimens of lung cancer patients. Cancer Res 1996;56: 2285-2288.

30 Melamed MR: Lung cancer screening results in the National Cancer Institute New York study. Cancer 2000;89:2356-2362.

- 31 Mao L, Hruban RH, Boyle JO, Tockman M, Sidransky D: Detection of oncogene mutations in sputum precedes diagnosis of lung cancer. Cancer Res 1994;54:1634-1637.

32 Kubik A, Polak J: Lung cancer detection: results of a randomized prospective study in Czechoslovakia. Cancer 1986;57:2427-2437.

- 33 Fontana RS, Sanderson DR, Woolner LB, Taylor WF, Miller WE, Muhm JR: Lung cancer screening: the Mayo program. J Occup Med 1986;28:746-750.

-34 Brett GZ: The value of lung cancer detection by six-monthly chest radiographs. Thorax 1968;23:414-420.

35 Oken MM, Hocking WG, Kvale PA, Andriole GL, Buys SS, Church TR, et al: Screening by chest radiograph and lung cancer mortality: the Prostate, Lung, Colorectal, and Ovarian (PLCO) randomized trial. JAMA 2011;306: 1865-1873.
36 Henschke CI, McCauley DI, Yankelevitz DF, Naidich DP, McGuinness G, Miettinen OS, et al: Early Lung Cancer Action Project: overall design and findings from baseline screening. Lancet 1999;354:99-105.

37 Mettler FA Jr, Huda W, Yoshizumi TT, Mahesh M: Effective doses in radiology and diagnostic nuclear medicine: a catalog. Radiology 2008;248:254-263.

38 Smith-Bindman R, Lipson J, Marcus R, Kim KP, Mahesh M, Gould R, et al: Radiation dose associated with common computed tomography examinations and the associated lifetime attributable risk of cancer. Arch Intern Med 2009;169:2078-2086.

39 Preston DL, Ron E, Tokuoka S, Funamoto S, Nishi N, Soda M, et al: Solid cancer incidence in atomic bomb survivors: 1958-1998. Radiat Res 2007;168:1-64.

40 van den Bergh KA, Essink-Bot ML, Bunge EM, Scholten ET, Prokop M, van Iersel CA, et al: Impact of computed tomography screening for lung cancer on participants in a randomized controlled trial (NELSON trial). Cancer 2008;113:396-404.

41 Henschke CI, Yankelevitz DF, Libby DM, Pasmantier MW, Smith JP, Miettinen OS: Survival of patients with stage I lung cancer detected on CT screening. N Engl J Med 2006; 355:1763-1771.

42 Bach PB: Overdiagnosis in lung cancer: different perspectives, definitions, implications. Thorax 2008;63:298-300.

43 Bach PB, Jett JR, Pastorino U, Tockman MS, Swensen SJ, Begg CB: Computed tomography screening and lung cancer outcomes. JAMA 2007;297:953-961.

44 Kubik AK, Parkin DM, Zatloukal P: Czech Study on Lung Cancer Screening: post-trial follow-up of lung cancer deaths up to year 15 since enrollment. Cancer 2000;89:2363-2368.

45 Marcus PM, Bergstralh EJ, Fagerstrom RM, Williams DE, Fontana R, Taylor WF, et al: Lung cancer mortality in the Mayo Lung Project: impact of extended follow-up. J Natl Cancer Inst 2000;92:1308-1316.

46 Veronesi G, Maisonneuve P, Bellomi M, Rampinelli C, Durli I, Bertolotti R, et al: Estimating overdiagnosis in low-dose computed tomography screening for lung cancer: a cohort study. Ann Intern Med 2012;157:776784 .

47 Kovalchik SA, Tammemagi M, Berg CD, Caporaso NE, Riley TL, Korch M, et al: Targeting of low-dose CT screening according to the risk of lung-cancer death. N Engl J Med 2013; 369:245-254.

48 Tammemagi MC, Katki HA, Hocking WG, Church TR, Caporaso N, Kvale PA, et al: Selection criteria for lung-cancer screening. N Engl J Med 2013;368:728-736.

49 Woloshin S, Schwartz LM, Black WC, Kramer BS: Cancer screening campaigns - getting past uninformative persuasion. $\mathrm{N}$ Engl J Med 2012;367:1677-1679.

50 American Lung Association: Providing guidance on lung cancer screening to patients and physicians. 2012. http://www.lung.org/lungdisease/lung-cancer/lung-cancer-screeningguidelines/lung-cancer-screening.pdf (accessed July 5, 2013).

51 American Society of Clinical Oncology: What to know: the ACCP and ASCO guideline on lung cancer screening. 2012. http:// www.cancer.net/sites/cancer.net/files/ what_to_know_lung_cancer_screening.pdf (accessed May 7, 2013).

52 Hansell DM, Bankier AA, MacMahon H, McLoud TC, Muller NL, Remy J: Fleischner Society: glossary of terms for thoracic imaging. Radiology 2008;246:697-722.

53 Austin JH, Muller NL, Friedman PJ, Hansell DM, Naidich DP, Remy-Jardin M, et al: Glossary of terms for CT of the lungs: recommendations of the Nomenclature Committee of the Fleischner Society. Radiology 1996;200: 327-331.

54 de Hoop B, van Ginneken B, Gietema H, Prokop M: Pulmonary perifissural nodules on CT scans: rapid growth is not a predictor of malignancy. Radiology 2012;265:611-616.

55 Gould MK, Donington J, Lynch WR, Mazzone PJ, Midthun DE, Naidich DP, et al: Evaluation of individuals with pulmonary nodules: when is it lung cancer? Diagnosis and management of lung cancer, 3 rd ed. American College of Chest Physicians evidence-based clinical practice guidelines. Chest 2013;143: e93S-e120S.

-56 Xu DM, van der Zaag-Loonen HJ, Oudkerk $\mathrm{M}$, Wang Y, Vliegenthart R, Scholten ET, et al: Smooth or attached solid indeterminate nodules detected at baseline CT screening in the NELSON study: cancer risk during 1 year of follow-up. Radiology 2009;250: 264-272.

57 MacMahon H, Austin JH, Gamsu G, Herold CJ, Jett JR, Naidich DP, et al: Guidelines for management of small pulmonary nodules detected on CT scans: a statement from the Fleischner Society. Radiology 2005;237:395400

58 Naidich DP, Bankier AA, Macmahon $\mathrm{H}$, Schaefer-Prokop CM, Pistolesi M, Goo JM, et al: Recommendations for the Management of Subsolid Pulmonary Nodules Detected at CT: A Statement from the Fleischner Society. Radiology 2012;266:304-317.

59 Godoy MC, Naidich DP: Subsolid pulmonary nodules and the spectrum of peripheral adenocarcinomas of the lung: recommended interim guidelines for assessment and management. Radiology 2009;253:606622.

60 Ahn MI, Gleeson TG, Chan IH, McWilliams AM, Macdonald SL, Lam S, et al: Perifissural nodules seen at CT screening for lung cancer. Radiology 2010;254:949-956.

61 Zhao Y, de Bock GH, Vliegenthart R, van Klaveren RJ, Wang Y, Bogoni L, et al: Performance of computer-aided detection of pulmonary nodules in low-dose CT: comparison with double reading by nodule volume. Eur Radiol 2012;22:2076-2084. 
62 Godoy MC, Kim TJ, White CS, Bogoni L, de Groot P, Florin C, et al: Benefit of computeraided detection analysis for the detection of subsolid and solid lung nodules on thin- and thick-section CT. AJR Am J Roentgenol 2013; 200:74-83.

63 Wormanns D, Ludwig K, Beyer F, Heindel W, Diederich S: Detection of pulmonary nodules at multirow-detector CT: effectiveness of double reading to improve sensitivity at standard-dose and low-dose chest CT. Eur Radiol 2005;15:14-22.

64 van't Westeinde SC, de Koning HJ, Thunnissen FB, Oudkerk M, Groen HJ, Lammers JW, et al: The role of the (1)(8)f-fluorodeoxyglucose-positron emission tomography scan in the Nederlands Leuvens Longkanker screenings Onderzoek lung cancer screening trial. J Thorac Oncol 2011;6:1704-1712.

65 Veronesi G, Bellomi M, Veronesi U, Paganelli G, Maisonneuve P, Scanagatta P, et al: Role of positron emission tomography scanning in the management of lung nodules detected at baseline computed tomography screening. Ann Thorac Surg 2007;84:959965, discussion 965-966.

66 Ashraf H, Dirksen A, Loft A, Bertelsen AK, Bach KS, Hansen H, et al: Combined use of positron emission tomography and volume doubling time in lung cancer screening with lowdose CT scanning. Thorax 2011;66:315-319.
67 Silva M, Sverzellati N, Manna C, Negrini G, Marchiano A, Zompatori M, et al: Long-term surveillance of ground-glass nodules: evidence from the MILD trial. J Thorac Oncol 2012;7:1541-1546.

68 Matsuguma H, Mori K, Nakahara R, Suzuki $\mathrm{H}$, Kasai T, Kamiyama Y, et al: Characteristics of subsolid pulmonary nodules showing growth during follow-up with CT scanning. Chest 2013;143:436-443.

69 Van Schil PE, Asamura H, Rusch VW, Mitsudomi T, Tsuboi M, Brambilla E, et al: Surgical implications of the new IASLC/ATS/ERS adenocarcinoma classification. Eur Respir J 2012;39:478-486.

70 Graham G, Blaha MJ, Budoff MJ, Rivera JJ, Agatston A, Raggi P, et al: Impact of coronary artery calcification on all-cause mortality in individuals with and without hypertension. Atherosclerosis 2012;225:432-437.

71 van de Wiel JC, Wang Y, Xu DM, van der Zaag-Loonen $\mathrm{HJ}$, van der Jagt EJ, van Klaveren RJ, et al: Neglectable benefit of searching for incidental findings in the DutchBelgian lung cancer screening trial (NELSON) using low-dose multidetector CT. Eur Radiol 2007;17:1474-1482.
72 Priola AM, Priola SM, Giaj-Levra M, Basso E, Veltri A, Fava C, et al: Clinical implications and added costs of incidental findings in an early detection study of lung cancer by using low-dose spiral computed tomography. Clin Lung Cancer 2013;14:139-148.

73 Kucharczyk MJ, Menezes RJ, McGregor A, Paul NS, Roberts HC: Assessing the impact of incidental findings in a lung cancer screening study by using low-dose computed tomography. Can Assoc Radiol J 2011;62:141-145.

74 National Center for Disease Control and Prevention: Smoking and Tobacco Use. 2013. http://www.cdc.gov/tobacco/tobacco control_programs/ (accessed August 8, 2013).

75 McMahon PM, Kong CY, Bouzan C, Weinstein MC, Cipriano LE, Tramontano AC, et al: Cost-effectiveness of computed tomography screening for lung cancer in the United States. J Thorac Oncol 2011;6:1841-1848.

76 Goulart BH, Bensink ME, Mummy DG, Ramsey SD: Lung cancer screening with lowdose computed tomography: costs, national expenditures, and cost-effectiveness. J Natl Compr Canc Netw 2012;10:267-275.

77 Pyenson BS, Sander MS, Jiang Y, Kahn H, Mulshine JL: An actuarial analysis shows that offering lung cancer screening as an insurance benefit would save lives at relatively low cost. Health Aff (Millwood) 2012;31: 770-779. 